This item was submitted to Loughborough's Institutional Repository (https://dspace.lboro.ac.uk/) by the author and is made available under the following Creative Commons Licence conditions.

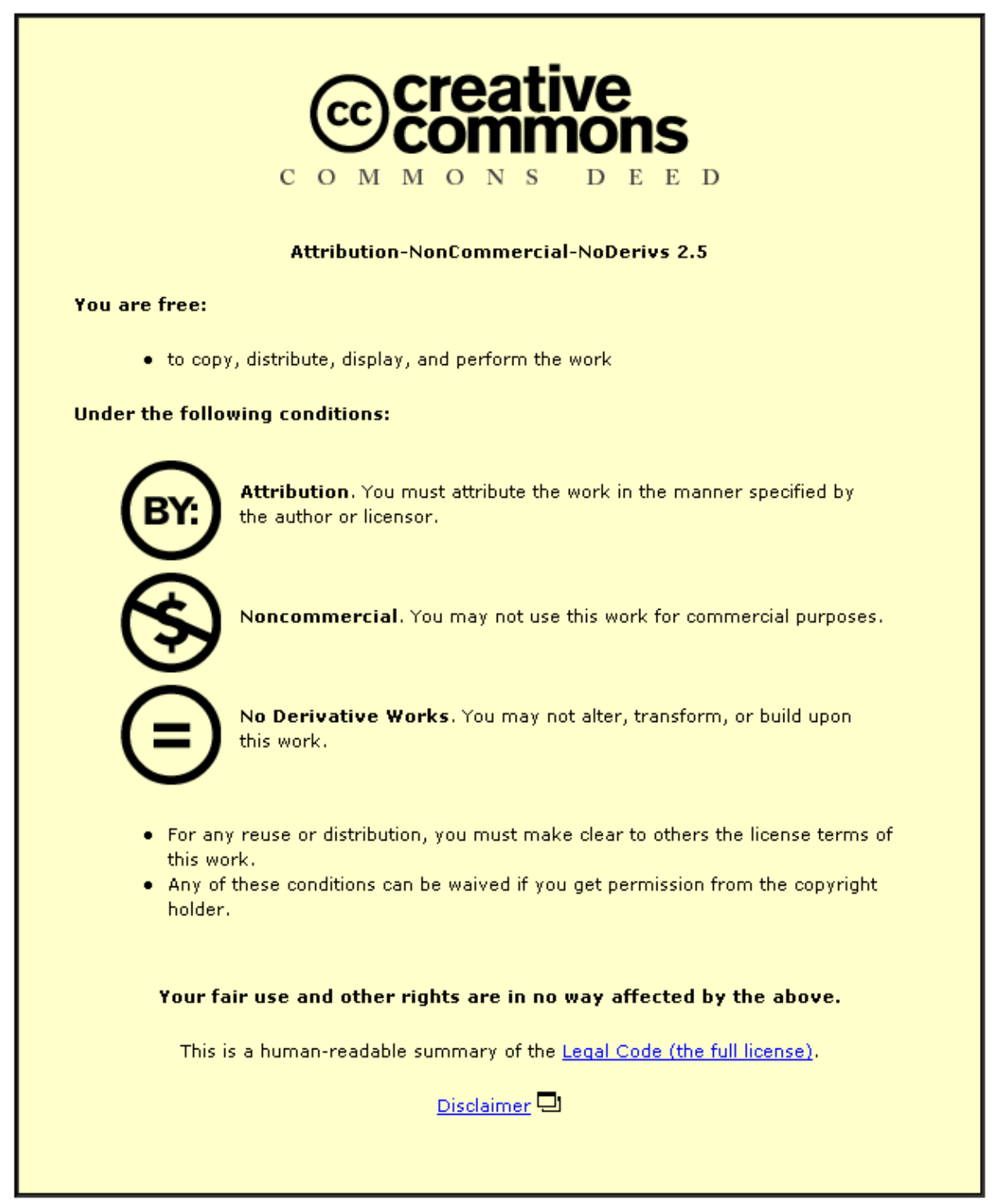

For the full text of this licence, please go to: http://creativecommons.org/licenses/by-nc-nd/2.5/ 


\title{
Protein destruction by a helium atmospheric pressure glow discharge: Capability and mechanisms
}

\author{
X. T. Deng, J. J. Shi, and M. G. Kong ${ }^{\text {a) }}$ \\ Department of Electronic and Electrical Engineering, Loughborough University, Leicestershire LE11 3TU, \\ United Kingdom
}

(Received 22 November 2006; accepted 9 February 2007; published online 4 April 2007)

\begin{abstract}
Biological sterilization represents one of the most exciting applications of atmospheric pressure glow discharges (APGD). Despite the fact that surgical instruments are contaminated by both microorganisms and proteinaceous matters, sterilization effects of APGD have so far been studied almost exclusively for microbial inactivation. This work presents the results of a detailed investigation of the capability of a helium-oxygen APGD to inactivate proteins deposited on stainless-steel surfaces. Using a laser-induced fluorescence technique for surface protein measurement, a maximum protein reduction of $4.5 \operatorname{logs}$ is achieved by varying the amount of the oxygen admixture into the background helium gas. This corresponds to a minimum surface protein of 0.36 femtomole $/ \mathrm{mm}^{2}$. It is found that plasma reduction of surface-borne protein is through protein destruction and degradation, and that its typically biphasic reduction kinetics is influenced largely by the thickness profile of the surface protein. Also presented is a complementary study of possible APGD protein inactivation mechanisms. By interplaying the protein inactivation kinetics with optical emission spectroscopy, it is shown that the main protein-destructing agents are excited atomic oxygen (via the 777 and $844 \mathrm{~nm}$ emission channels) and excited nitride oxide (via the 226, 236, and $246 \mathrm{~nm}$ emission channels). It is also demonstrated that the most effective protein reduction is achieved possibly through a synergistic effect between atomic oxygen and nitride oxide. This study is a useful step toward a full confirmation of the efficacy of APGD as a sterilization technology for surgical instruments contaminated by prion proteins. (C) 2007 American Institute of Physics. [DOI: 10.1063/1.2717576]
\end{abstract}

\section{INTRODUCTION}

While the use of ionized gases as a microbiocidal agent was first reported in 1968, their potential as a genuine sterilization technology has attracted serious and widespread attention only over the past 10 years. One of the key stimuli has been the increasing inadequacy of conventional hospital procedures for inactivation of prion proteins and for sterilization of tubular instruments such as endoscopes. Widely regarded as the etiologic agent of spongiform neurodegenerative pathologies such as bovine spongiform encephalopathy (BSE), scrapie, and Creutzfeldt-Jakob diseases (CJD), ${ }^{1}$ prion proteins are extremely resistant to all current hospital decontamination procedures including autoclaving, ionizing radiation, and formaldehyde. ${ }^{2}$ As a consequence, there is an urgent and fundamental need for new and effective prion inactivation strategies. The advent of atmospheric pressure glow discharges (APGD) (Ref. 3) offers an exciting opportunity for prion inactivation with the advantage of minimizing the environmental burden of wet chemistry. Operating typically in open air without a vacuum chamber, APGD produce large fluxes of energetic and highly reactive plasma species such as UV photons, charged particles, and reactive oxygen species, all of which can potentially cause damage to both microorganisms and proteinaceous matters. So far, the bio-decontamination effect of APGD has been examined almost exclusively for microbial inactivation, in which case

${ }^{a)}$ Corresponding author; electronic mail: m.g.kong@lboro.ac.uk they have been shown comprehensively to be capable of inactivating wide-ranging microorganisms including the vegetative cells of yeast and bacteria, viruses, bacterial spores, and even biofilm-forming bacteria. ${ }^{4-8}$ A great deal has been learned about how APGD inactivation depends on the plasma operating conditions $s^{9,10}$ and the physiology of the microbial population. ${ }^{11,12}$ These studies have helped establish convincingly the microbiocidal capability of APGD. Today, atmospheric pressure glow discharges are widely regarded as one of the most exciting technology platforms that is likely to impact profoundly on the bio-decontamination practice and policy in both the healthcare service and the food industry. ${ }^{4,8}$

Much less known is the capability of APGD for inactivation and destruction of proteinaceous matters, ${ }^{13,14}$ even though surgical instruments are usually contaminated by both bacteria and proteins. This lack of understanding is of great concern, given the considerable risks of prion contamination of surgical instruments to many patients, particularly those who require neurosurgery. For the APGD technology to become a genuine sterilization solution for the healthcare service, it is essential to study and develop its capability for protein inactivation. In this article, we present a detailed investigation of protein destruction using an APGD jet in helium-oxygen flow in order to (a) establish the protein inactivation capability of atmospheric pressure glow discharges and (b) offer insights into possible mechanisms of plasma protein destruction. In Sec. II, materials and methods 


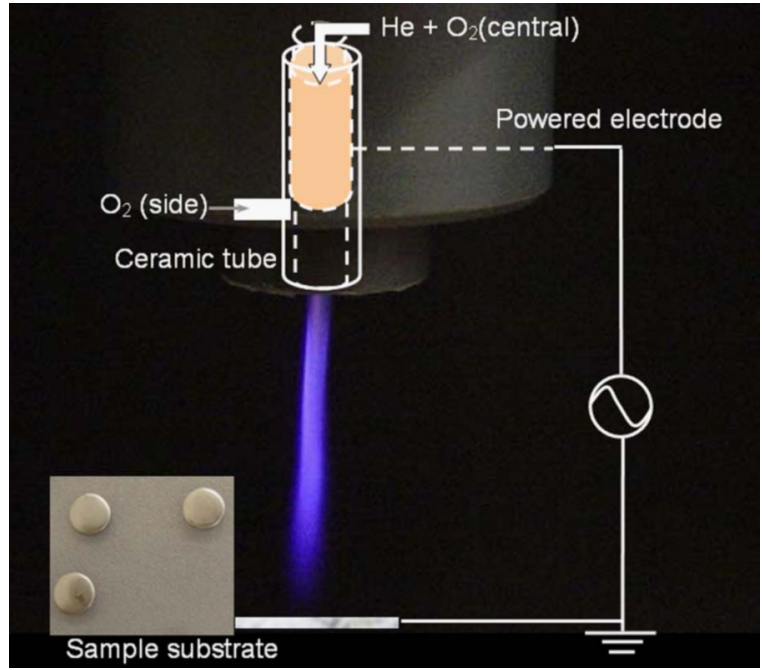

FIG. 1. (Color online) Schematic of the atmospheric-pressure $\mathrm{He}-\mathrm{O}_{2}$ plasma jet with its connection to the external circuit and an image of the stainlesssteel disk samples. The APGD jet is struck inside a ceramic tube and the ceramic tube is enclosed inside a PTFE hosting unit (shown as the gray object in the background).

used in this study are reviewed with an emphasis on both the APGD jet system employed and the measurement of protein deposited on solid surfaces. This is followed in Sec. III by a systematic investigation of protein inactivation using protein reduction kinetics analogous to those used in microbial inactivation. ${ }^{15}$ Given the current interests in and uncertainties of plasma inactivation mechanisms, we also include in Sec. IV a first study of possible protein-inactivating plasma species with a view to ultimately unravel APGD protein inactivation mechanisms. Our strategy is to exploit possible correlations of the protein reduction kinetics to the relative densities of key plasma species. Finally, in Sec. V, findings of this study are summarized and important issues for the future development of the APGD protein inactivation technology are discussed.

\section{MATERIALS AND METHODS}

The atmospheric pressure plasma system used in our study was a cold atmospheric plasma jet struck inside a helium-oxygen channel and ejected into the ambient air. As shown in Fig. 1, the gas channel was formed by flushing a helium-oxygen gas mixture through a ceramic tube around which a metallic strip of $10 \mathrm{~mm}$ wide was wrapped as the powered electrode. The inside and outside diameters of the ceramic tube were 1.5 and $2 \mathrm{~mm}$, respectively, and the ceramic tube was enclosed inside a PTFE hosting unit. The grounded electrode was a dielectrically insulated metal plate, and was placed downstream about 2-20 $\mathrm{mm}$ away from the nozzle of the ceramic tube. Depending on the applied voltage and the gas flow rate, the plasma jet was about $10-40 \mathrm{~mm}$ in length and $2-3 \mathrm{~mm}$ in diameter. Details of the plasma jet have been given elsewhere. ${ }^{17,18}$ For this study, the APGD jet was energized with a sinusoidal voltage source with a peak voltage of 4-8 $\mathrm{kV}$ and an excitation frequency of 30-45 kHz. Helium (99.9999\%) was used as the working gas with its flow rate fixed at $5 \mathrm{slm}$, either alone or mixed with oxy- gen gas of varying flow rates. An auxiliary oxygen flow, marked as the side $\mathrm{O}_{2}$ in Fig. 1, was sometimes introduced into the ceramic tube at a downstream entrance from the powered electrode. For experiments reported here, the entrance of the side $\mathrm{O}_{2}$ flow was fixed at $3 \mathrm{~mm}$ away from the nozzle-side edge of the powered electrode. The purpose of this side $\mathrm{O}_{2}$ flow was to make available more ground-state oxygen molecules. The APGD jet system separated the helium-dominating plasma generation region from the treatment region where air chemistry was introduced. Because of its capability for more active plasma chemistry, it was selected instead of one with the usual parallel-plate configuration.

At present, standard hospital sterilization procedures such as autoclaving are used in medical sterilization units to clean and sterilize surgical instruments. Autoclaving procedures are very effective in washing off large quantities of tissue fragments and other organic matters left on medical devices following surgeries. Unfortunately, they often leave small amount of protein residues on surgical instruments, and the autoclaved protein residues tend to attach tightly to the surfaces of the instruments. ${ }^{16}$ These protein residues are the source of cross contamination. ${ }^{1}$ Therefore, any plasma protein inactivation strategy is perhaps best introduced as an additional step in the decontamination process after the autoclaving cycle. Given that protein deposits following the autoclaving procedure tend to be localized and their size tends to be small, ${ }^{16}$ the APGD jet of Fig. 1 is appropriate. Given the highly infectious nature of prion proteins, our study employed bovine serum albumin (BSA) as a model protein, purchased from Sigma-Aldrich (Dorset, UK). To model surgical instruments, stainless-steel disks of $6 \mathrm{~mm}$ diameter were made and used as the sample substrate. Examples of such disks are shown in the inset of Fig. 1. Quantities of BSA, up to $20 \mu \mathrm{l}$ per droplet, were diluted in distilled water and then their droplets were deposited on stainless-steel surfaces to dry at $50{ }^{\circ} \mathrm{C}$ before plasma treatment. The diameter of the BSA deposit on the stainless-steel disk was typically $5 \mathrm{~mm}$. All data of plasma protein inactivation experiments were in triplicate from three independent experiments.

The discharge current and the applied voltage were measured by a wideband current probe (Tektronix P6021) and a wideband voltage probe (Tektronix P6015A), respectively, and their waveforms were recorded on a digital oscilloscope (Tektronix TDS 3034B). Optical emission spectrum from the APGD jet was obtained using a spectrometer system (Andor Shamrock) with a focal length of $0.3 \mathrm{~m}$ and a grating of 600 grooves $/ \mathrm{mm}$. The spectral measurement was taken from the contact point of the plasma jet with the sample surface. To assess the efficacy of the plasma protein inactivation, it is important to quantitatively measure surface-borne protein, before and after plasma treatment. While standard biology laboratory methods, such as spectrophotometers, can be used to reliably measure protein in suspension, they cannot be used directly for measurement of surface-borne protein. Since no established devices are commercially available for measurement of surface-borne protein, we employed a laserinduced fluorescence technique (LIF), ${ }^{19}$ and developed an 

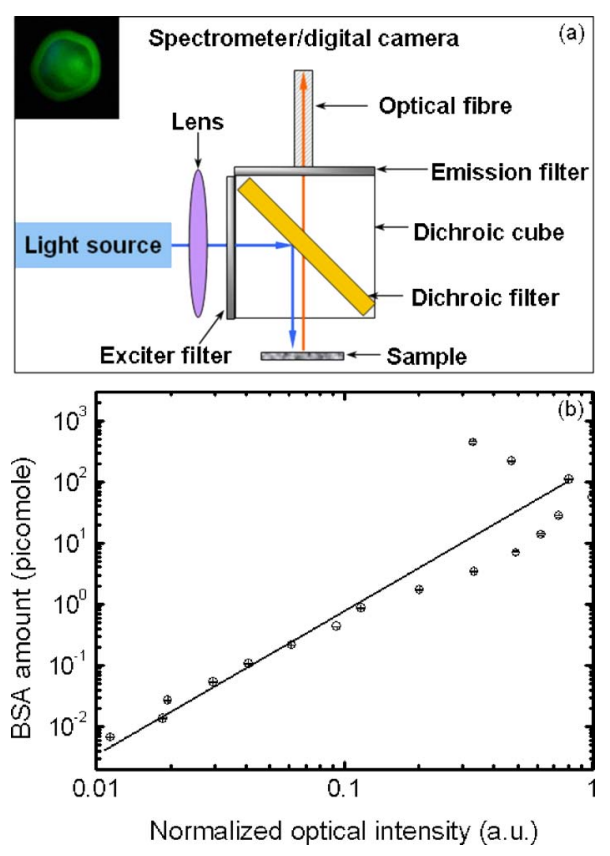

FIG. 2. (Color online) (a) Schematic of the laser-induced fluorescence (LIF) system for surface protein measurement with the inset being the fluorescence image of a protein contaminated stainless-steel sample; (b) the linear dependence of the surface protein on the fluorescence intensity.

optical system to quantitatively detect proteinaceous matters on stainless-steel surfaces. Specifically, the BSA sample was labeled with FITC (fluorescein isothiocyanate) through covalent binding. ${ }^{19,20}$ With appropriate light irradiation, the FITClabeled protein fluoresces and the fluorescence intensity can be correlated with the amount of the surface protein thus offering a means of protein detection. The schematic of the laser-induced fluorescence apparatus is shown in Fig. 2(a), with the inset being an example of the FITC-labeled BSA under laser irradiation. The optimum excitation wavelength for the fluorescence was $488 \mathrm{~nm}$ and the maximum fluorescence emission was around $530 \mathrm{~nm}$.

The excitation signal was provided by a laser-emitting diode (Ocean Optics LS 475) with a signal bandwidth of 460-490 nm. Wavelength filtering was found critical to reduce signal interference and extend the detection limit. Interference from reflected excitation light from the stainless-steel surface was effectively removed by the emission filter (Thorlabs, FEL0500). Another source of interference was Raman scattering, ${ }^{20}$ particularly when the deposited protein was at a sufficiently low level. This was generated in the excitation fiber at about $430 \mathrm{~nm}$ and reflected by the stainless-steel surface into the emission fiber at about $470 \mathrm{~nm} .{ }^{20}$ However the reflected Raman scattering signal had a long tail extending into 480-580 $\mathrm{nm}$ and overlapping the fluorescence signal at $530 \mathrm{~nm}$. To overcome this, we employed both the dichroic filter (a $505 \mathrm{~nm}$ Nikon filter) and the exciter filter (Laser2000, FF506-Ex04-25) to minimize the Raman scattering signal, similar to the technique reported in the literature. ${ }^{20}$ With these wavelength filters, a linear correlation was found to exist between the surface-deposited BSA protein and the fluorescence intensity from 55 picomole to 6.7 femtomole as shown in Fig. 2(b). Given that the surface area of the BSA deposit was about $4.9 \mathrm{~mm}^{2}$, the above minimum suggests a detection limit of 1.4 femtomole $/ \mathrm{mm}^{2}$ or 1.4 $\times 10^{9}$ molecules $/ \mathrm{mm}^{2}$. It is worth mentioning that the fluorescence quenching of molecules in close proximity to metal surfaces was considered, with the main effect being a reduction of about $60 \%$ in the quantum yield of the fluorescence compared to that in solution. ${ }^{20}$ To ensure that this has been accounted for accurately, we calibrated the results of the LIF method in Fig. 2 against those obtained from a photon counting fluorescence spectrometer (Jobin-Yvon-Horiba Fluoromax-Pro). This has confirmed that the LIF system in Fig. 2(a) offers a quantitative means to measure surfaceborne protein reliably and accurately.

\section{PROTEIN REDUCTION KINETICS}

BSA-laden stainless-steel samples were plasma-treated over different periods of time after which surface proteins were measured using the laser-induced fluorescence technique of Fig. 2. The relationship of residual BSA proteins on the stainless-steel surface to the plasma treatment time is effectively the protein reduction kinetics, very similar to the microbial inactivation kinetics. ${ }^{15}$ Effects of different plasma parameters were studied, of which the most important were (1) the peak applied voltage, $V_{p}$; (2) the distance from the nozzle of the ceramic tube to the sample, $d_{n s}$; (3) the flow rate of the oxygen gas added to the helium flow through the powered electrode; and (4) the flow rate of an auxiliary oxygen gas introduced downstream from the powered electrode (see Fig. 1). These parameters were considered because they are likely to significantly affect the plasma chemistry at the point of the protein sample and hence protein reduction. For example, the applied voltage influences directly the level of the gas ionization and hence the production of reactive plasma species. As all plasma species have a finite life, the nozzle-to-sample distance is likely to alter the concentration of plasma species at the sample point and hence their efficiency for protein reduction. On the other hand, reactive oxygen species are known to play a dominant role for plasma microbial inactivation. ${ }^{21}$ Therefore, it is desirable to add the oxygen gas into the working gas to provide an additional source for reactive oxygen species. For future reference, the oxygen gas added to the He flow through the powered electrode is referred to as the central $\mathrm{O}_{2}$ flow and the auxiliary oxygen gas introduced downstream from the powered electrode is referred to as the side $\mathrm{O}_{2}$ flow. The central $\mathrm{O}_{2}$ flow is introduced for direct ionization within the ceramic tube by the applied voltage, and the side $\mathrm{O}_{2}$ flow is intended as an additional oxygen source to be exited and/or ionized by energetic and long-life helium metastables. Atomic helium metastables $\mathrm{He}\left(2 s{ }^{1} S_{0}\right)$ and $\mathrm{He}\left(2 s^{3} S_{1}\right)$ are produced with excitation energy of 20.61 and $19.82 \mathrm{eV}$, respectively, both greater than the ionization energy of oxygen at $13.62 \mathrm{eV}$. So, He $\left(2 s{ }^{1} S_{0}\right)$ and He $\left(2 s^{3} S_{1}\right)$ metastables are capable of ionizing ground-state oxygen molecules in the ambient air. Here, we consider in turn the effects of the above four different parameters. 


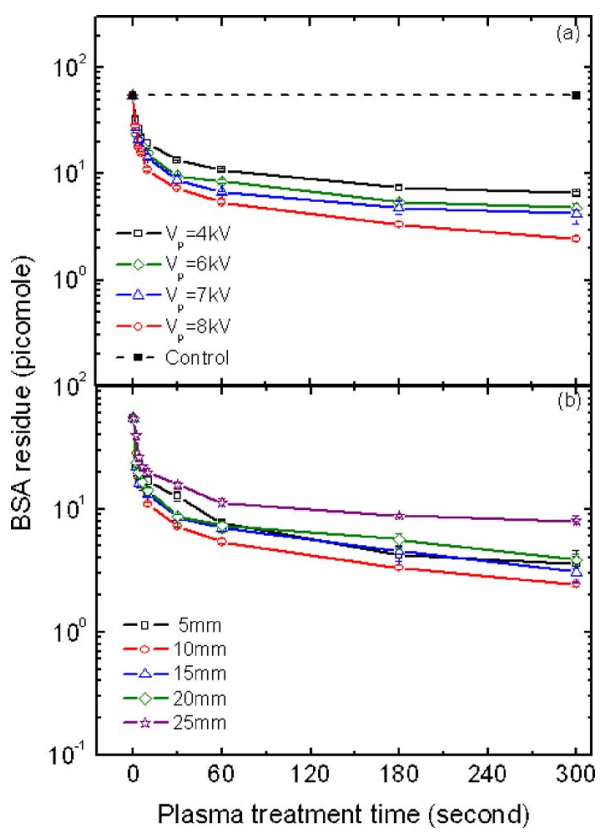

FIG. 3. (Color online) Protein reduction kinetics with the APGD jet in a 5 slm pure helium flow (a) at $d_{n s}=10 \mathrm{~mm}$ and different applied voltages; and (b) at different tube-to-sample distances and $V_{p}=8 \mathrm{kV}$. The control experiment in (a) was done in a 5 slm helium flow with the plasma off and $d_{n s}$ $=10 \mathrm{~mm}$.

\section{A. Effects of the applied voltage and the nozzle-sample distance}

With $d_{n s}$ fixed at $10 \mathrm{~mm}$, pure helium was first used as the working gas and fed through the ceramic tube for ionization. As the applied voltage was increased, the plasma jet became brighter and its length became longer (images not shown). This suggests that larger fluxes of excited species were produced at higher levels of the applied voltage. Figure 3(a) shows protein reduction kinetics at different peak values of the applied voltage, for which the control experiment was performed in a $5 \mathrm{slm}$ helium flow with the plasma off. It is evident that the $5 \mathrm{slm}$ helium flow with the APGD turned off led to negligible protein removal. It is also evident that greater protein reduction was achieved at larger applied voltage. This is in a broad agreement with the large fluxes of excited plasma species produced at larger applied voltage. For our experiments, the maximum peak applied voltage was limited at $8 \mathrm{kV}$, beyond which the plasma jet started to heat up the protein sample. Figure 3(b) shows protein reduction kinetics at different values of $d_{n s}$ with $V_{p}=8 \mathrm{kV}$ and again pure helium as the working gas. Protein reduction is seen to be the most effective at a nozzle-to-sample distance of 10 $\mathrm{mm}$. As oxygen-exciting and/or oxygen-ionizing agents are ejected out of the ceramic tube by the plasma jet, they are likely to reduce in both their energy and concentrations after a critical nozzle-to-sample distance. It is therefore conceivable that protein reduction becomes less effective when $d_{n s}$ is too long. If the nozzle-to-sample distance is too short, on the other hand, ground-state oxygen molecules outside the tube nozzle are likely to be pushed away from the sample region by the high-speed ejecting plasma jet. This reduces the amount of available oxygen molecules that can be excited and/or ionized near the protein sample, thus compromising
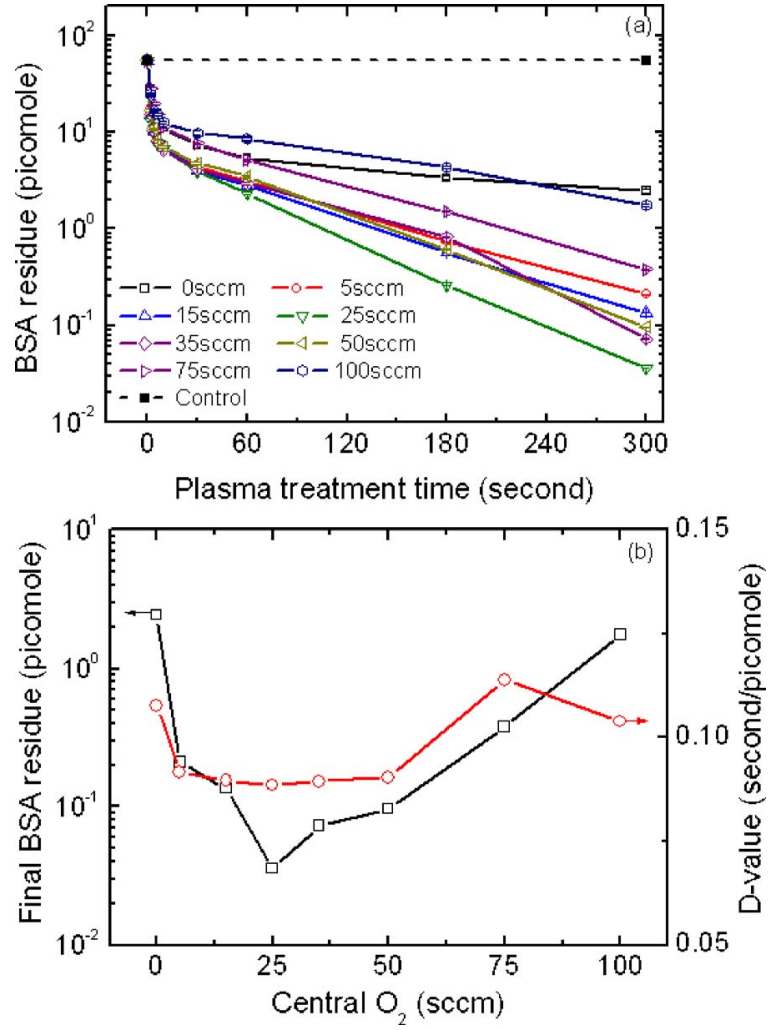

FIG. 4. (Color online) Effects of the central $\mathrm{O}_{2}$ admixture as shown in (a) protein reduction kinetics and (b) the $\mathrm{O}_{2}$ flow rate dependence of the BSA residue and the $D$ value. No side $\mathrm{O}_{2}$ flow. The control experiment in (a) was done in a $\mathrm{He}-\mathrm{O}_{2}$ flow $\left(\mathrm{He}: 5 \mathrm{slm} ; \mathrm{O}_{2}: 25 \mathrm{sccm}\right)$ with the plasma off and $d_{n s}=10 \mathrm{~mm}$.

protein reduction. From Fig. 3, the optimum plasma condition appears to be $V_{p}=8 \mathrm{kV}$ and $d_{n s}=10 \mathrm{~mm}$. These are used for our studies below.

\section{B. Effects of the central $\mathrm{O}_{2}$ flow rate}

With the rate of the central oxygen flow increasing from zero to $100 \mathrm{sccm}$, the protein reduction kinetics changed considerably as shown in Fig. 4(a). The control experiment included in Fig. 4(a) was performed with the plasma off in an un-ionized helium-oxygen flow for which the helium and oxygen flow rates were at $5 \mathrm{slm}$ and $25 \mathrm{sccm}$, respectively. It is shown in Fig. 4(a) that the un-ionized $\mathrm{He}-\mathrm{O}_{2}$ flow had a negligible effect on protein removal. The most effective protein reduction is seen to occur at a central $\mathrm{O}_{2}$ flow rate of 25 $\mathrm{sccm}$. To compare different cases more clearly, we introduce two quantification parameters of (a) the plasma treatment time to achieve one $\log$ protein reduction, or the $D$ value, ${ }^{15}$ at the beginning of the plasma treatment; and (b) the final amount of protein residue after $300 \mathrm{~s}$ of the plasma treatment. These represent the initial protein reduction rate and the total accumulated protein reduction, respectively. As the $D$ value is calculated at $t=0$, it is likely to reflect directly the effect of the most lethal plasma species. Figure 4(b) suggests that the shortest $D$ value was $88 \mathrm{~ms}$ per picomole over a wide flow rate range of 5-50 sccm, suggesting the persistence of the most lethal plasma species in this flow rate range. 
Figure 4(b) also suggests that the lowest final protein residue was 35 femtomoles at a central $\mathrm{O}_{2}$ flow rate of 25 sccm. The existence of an optimal flow rate may be interpreted as follows. When the central $\mathrm{O}_{2}$ flow rate is too low, less than $25 \mathrm{sccm}$ in our case, the available oxygen molecules in the $\mathrm{He}-\mathrm{O}_{2}$ working gas are insufficient for the maximum oxygen excitation and/or ionization to be reached at a given peak applied voltage of $V_{p}=8 \mathrm{kV}$. As a result, the production of reactive oxygen species is not the most effective and the resulting protein reduction is also below optimal. If the central $\mathrm{O}_{2}$ flow rate is too large, more than $25 \mathrm{sccm}$ in our case, the oversupply of available ground-state oxygen molecules increases the breakdown voltage of the $\mathrm{He}-\mathrm{O}_{2}$ working gas and so decreases the level of the gas ionization at $V_{p}=8 \mathrm{kV}$. This compromises the production of reactive oxygen species at the sample point and in turn reduces the efficiency of protein inactivation. It is conceivable that the final protein residue is the combined results of many different plasma species accumulated over a given period of time. The observation in Fig. 4(b) of its clear minimum at $25 \mathrm{sccm}$ suggests that some of the less lethal plasma species may have worked synergistically with the most lethal species to cause a sharp protein reduction at $25 \mathrm{sccm}$.

\section{Effects of the side $\mathrm{O}_{2}$ flow}

The clear protein reduction with pure helium in Fig. 3 suggests that it is possible for reactive oxygen species to be produced outside the plasma generation zone in a downstream region where energetic helium metastables and charged particles excite and/or ionize ground-state oxygen molecules in the ambient air. It is therefore of interest to examine whether a greater protein reduction can be achieved by making available of more ground-state oxygen molecules at a downstream point. To this end, the side $\mathrm{O}_{2}$ flow was introduced and the resulting protein reduction kinetics obtained is shown in Fig. 5(a). Again, the $D$ value and the final protein residue are used here to quantify the effects of the side $\mathrm{O}_{2}$ flow. As shown in Fig. 5(b), the introduction of the side oxygen flow tends to compromise protein reduction and is in general undesirable. Although the exact reason is unclear for the observed negative results, it is possible that the side-fed oxygen gas may have overly diluted the concentrations of helium metastables and/or electrons. Further parametric studies are of interest, but are best carried out in a separate study.

\section{Plasma conditions for effective protein reduction}

Together with the earlier discussion of Fig. 4, it is clear that the optimal protein reduction is achieved with no side $\mathrm{O}_{2}$ flow and with a central $\mathrm{O}_{2}$ flow at $25 \mathrm{sccm}$. Figures 4 and 5 have therefore clearly established the protein reduction capability of atmospheric pressure glow discharges in general and the APGD jet of Fig. 1 in particular. The minimum protein residue after plasma treatment in our experiments was 35 femtomoles or $7.1 \mathrm{femtomoles} / \mathrm{mm}^{2}$. It is possible to reduce this further using, for example, different gases, ${ }^{6,7,20}$ different temporal features of the electrical excitation, ${ }^{10,18,22}$ and indeed different APGD configurations. ${ }^{5,23}$ A related issue is
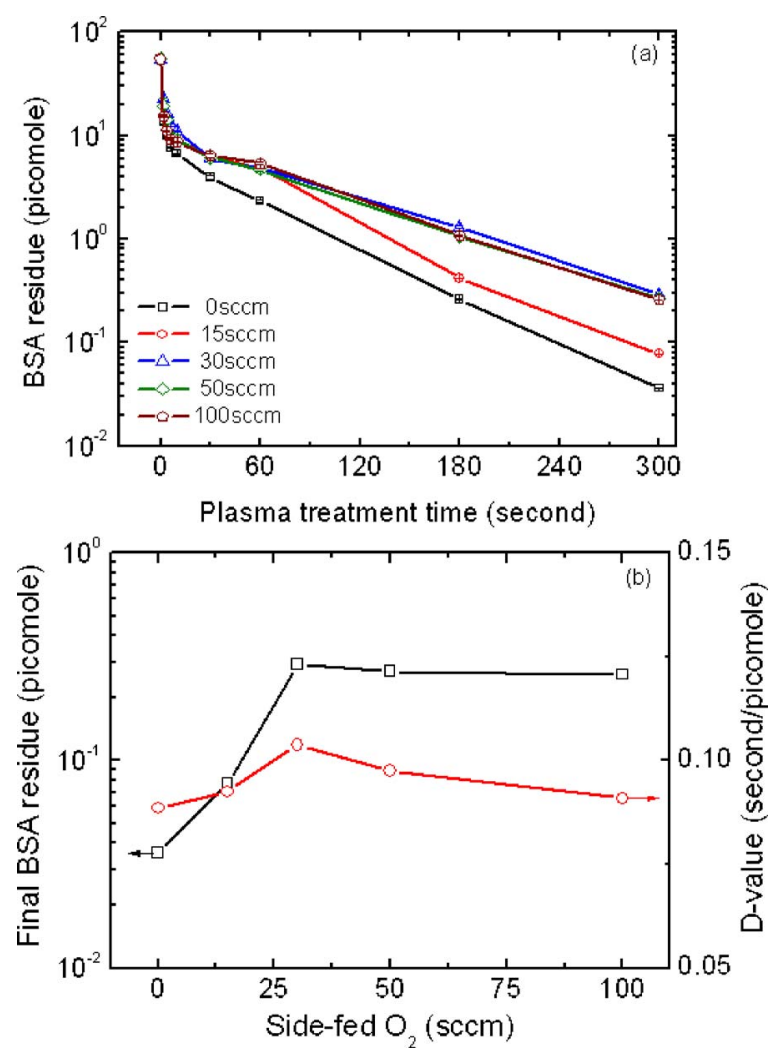

FIG. 5. (Color online) Effects of the side $\mathrm{O}_{2}$ admixture as shown in (a) protein reduction kinetics and (b) the $\mathrm{O}_{2}$ flow rate dependence of the BSA residue and the $D$ value. The central $\mathrm{O}_{2}$ flow rate is fixed at $25 \mathrm{sccm}$ and the helium rate is at $5 \mathrm{slm}$.

whether a minimum protein residue of 7.1 femtomoles $/ \mathrm{mm}^{2}$ is acceptable for plasma-treated surgical instruments to be reused, and indeed what the acceptable minimum of protein residue may be. At present, these questions remain unanswered and the ultimate answer lies in a thorough bioassay study. It is however important to emphasize that protein infectivity depends not only on the amount of protein residue but also their biological characters. In a very recent study, we used electrophoresis experiments to demonstrate that protein matters remaining on their substrate surface following plasma treatment sustained considerable degradation. ${ }^{14}$ Therefore, even when plasma treatment cannot completely remove all proteinaceous matters from stainless-steel surfaces, the plasma-treated but unremoved proteins are likely to have a compromised integrity and perhaps a reduced infectivity, thus posing less risk than untreated proteins.

\section{PROTEIN REDUCTION MECHANISMS}

Protein reduction kinetics in Figs. 4(a) and 5(a) exhibits two phases-an initial rapid reduction and then a much slower reduction over an extended period of time. Under plasma conditions not discussed here, it is possible for an additional phase to appear between the initial phase and the final extended phase, thus exhibiting three phases. These phases appear to represent different modes of protein inactivation. Although this is very similar to microbial inactivation by APGD, ${ }^{15}$ the underlying reasons may not necessarily be the same. In the case of microbial inactivation, different 


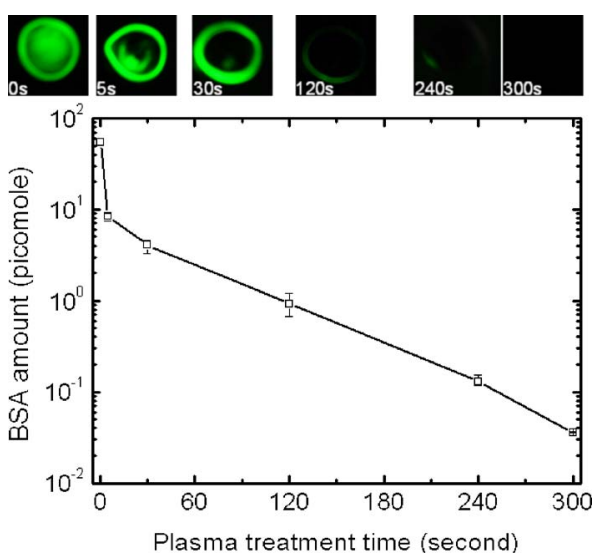

FIG. 6. (Color online) Reduction kinetics of surface protein with the fluorescence images of the BSA-contaminated sample after 0, 5, 30, 120, 240, and $300 \mathrm{~s}$ of the APGD treatment. Conditions are $V_{a}=8 \mathrm{kV}$, the central $\mathrm{O}_{2}$ flow rate $=25 \mathrm{sccm}$, and the side $\mathrm{O}_{2}$ flow rate $=0 \mathrm{sscm}$.

modes of the microorganism resistance to plasma treatment can be a result of many different factors including the cell wall, ${ }^{4,6}$ phase of cell growth, ${ }^{12}$ and cell stacking. ${ }^{17}$ To understand the factors that contribute to the essentially biphasic kinetics of plasma protein reduction, we took fluorescence images of the FITC-labeled protein sample after different plasma treatment times. These are shown in Fig. 6 together with the protein reduction curve under the optimal conditions of $V_{p}=8 \mathrm{kV}$, a central $\mathrm{O}_{2}$ flow rate of $25 \mathrm{sccm}$, and a side $\mathrm{O}_{2}$ flow rate of $0 \mathrm{sccm}$. It is clear that the first $30 \mathrm{~s}$ of the plasma treatment were effective in removing the central region of the protein deposit. By contrast, the edge region of the protein deposit was far more difficult to remove and required further $270 \mathrm{~s}$ to substantially reduce the protein fluorescence signal. Close evaluation of the protein-laden stainless-steel disks revealed that the protein deposit was thin in its middle and thick in its edge (data not shown). Therefore, the biphasic character of the protein reduction kinetics is largely related to the thickness profile of the surface protein, similar to the microbial loading effect in plasma bacterial inactivation. ${ }^{11}$ One important implication of this finding is that a more efficient protein removal would be achieved if localized protein deposits on surgical instruments could be identified, using fluorescence techniques for example, for them to be specifically treated by the APGD jet.

Efficiency of protein reduction can be improved if the most lethal plasma species are identified. Factors responsible for protein reduction usually fall into five categories, namely (1) heat; (2) charged particles; (3) intense electric field; (4) UV photons; and (5) neutral reactive species, all commonly present in a gas discharge. These are known to contribute to microbial inactivation by APGD, ${ }^{21}$ and could potentially be responsible for protein reduction observed in Figs. 4 and 5. For example, the electric field in the sheath region of APGD can be as high as $100 \mathrm{kV} / \mathrm{cm}$ (Ref. 24) and may cause an irreversible damage to protein. ${ }^{25,26}$ However, with our APGD jet configuration, the protein reduction took place at a downstream point away from the plasma generation region and away from the APGD sheath region. Therefore, the contribution of the electric field is likely to be very small, though this has yet to be confirmed by direct measurement of the electric

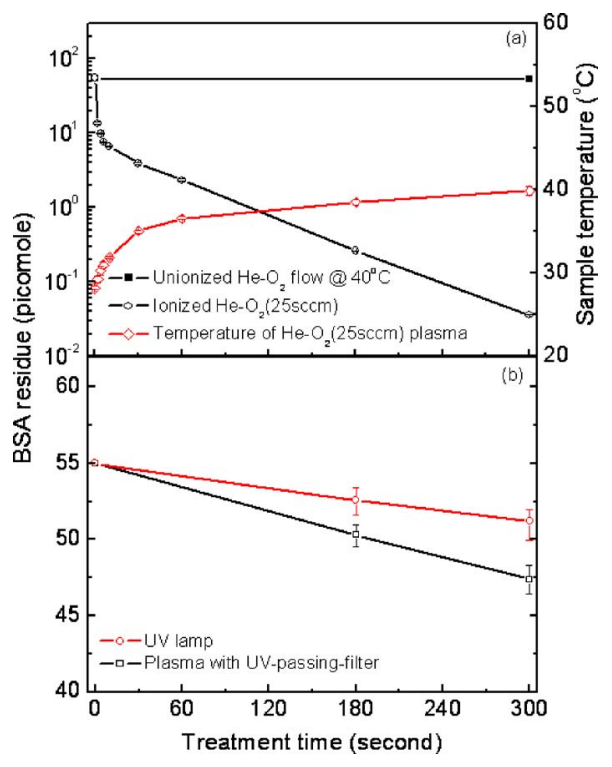

FIG. 7. (Color online) (a) Protein reduction (black, circles) and the sample temperature (red, diamonds) as a function of the treatment time, together with the control of an un-ionized $\mathrm{He}-\mathrm{O}_{2}$ gas flow (black, squares); (b) protein reduction by UV photons produced by the plasma jet (black, squares) and a commercial UV lamp (red, circles).

field. To establish the contribution of heat and UV photons, we measured the temperature of the protein substrate as a function of plasma treatment time for the $\mathrm{He}+\mathrm{O}_{2}$ case with the central $\mathrm{O}_{2}$ flow rate being $25 \mathrm{sccm}$. As shown in Fig. 7 (a), the highest temperature reached was $40{ }^{\circ} \mathrm{C}$. To show its effect, we used an un-ionized $\mathrm{He}+\mathrm{O}_{2}$ flow (with the electrical power switched off) to treat a protein sample that was kept at a constant temperature of $40{ }^{\circ} \mathrm{C}$. No protein reduction was observed after $300 \mathrm{~s}$ of the treatment. With the sample unheated but with the $\mathrm{He}+\mathrm{O}_{2}$ gas ionized at $V_{p}$ $=8 \mathrm{kV}$, surface-borne BSA was found to reduce from 55 picomoles to 35 femtomoles over $300 \mathrm{~s}$. It is therefore clear that heat generated in our APGD jet had a negligible protein reduction effect. To investigate the effect of UV, we employed a commercial UV lamp (Ultra Violet Products Ltd, Cambridge, UK) with its wavelength at $254 \mathrm{~nm}$ and its power consumption at about $2 \mathrm{~W}$. After $300 \mathrm{~s}$ of the UV treatment, protein reduction was a very limited 3 picomoles from an initial 55 picomoles as shown in Fig. 7(b). We also employed an UV passing glass (Thorlabs) to cover the protein sample so that only light of $170-400 \mathrm{~nm}$ could pass through. ${ }^{21}$ The achieved reduction was about 7 picomole over $300 \mathrm{~s}$, still much smaller than the $3 \log$ reduction seen in Fig. 7(a). It is therefore clear that both heat and UV photons were unlikely to be responsible for a significant protein reduction in our experiments.

Excited-states reactive plasma species can be detected with optical emission spectroscopy. Figure 8 shows the optical emission spectrum of the APGD jet of Fig. 1, taken near the sample point and at a central $\mathrm{O}_{2}$ flow rate of $25 \mathrm{sccm}$. It is clear that they exhibit strong emission lines of helium species as well as UV, OH radicals, oxygen species, and nitrogen species. The presence of the oxygen and nitrogen species was a result of the fact that the APGD was ejected into the ambient air where its energetic electrons and meta- 


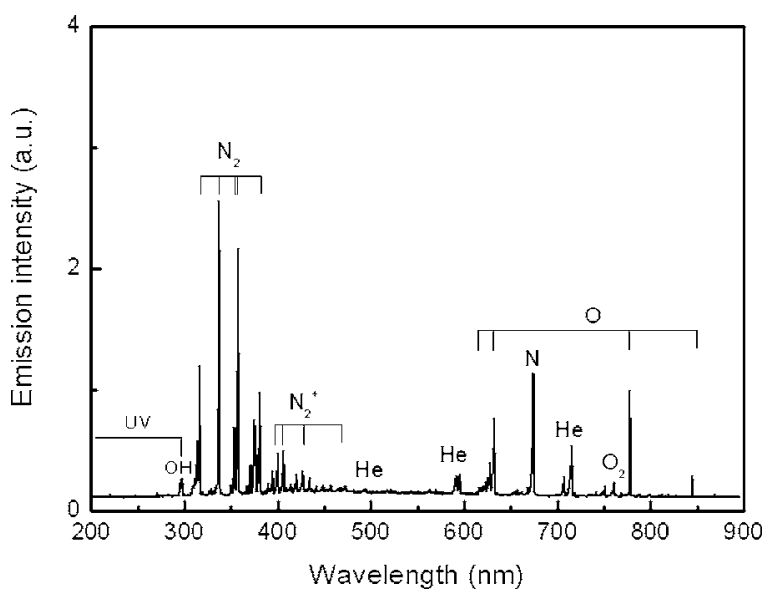

FIG. 8. Optical emission spectra of the APGD jet near the sample point with $V_{a}=8 \mathrm{kV}$, no side $\mathrm{O}_{2}$ flow, and a central oxygen flow rate of $25 \mathrm{sccm}$.

stables ionized and excited air molecules. ${ }^{18,21}$ The three strongest helium lines are at 587, 668, and $706 \mathrm{~nm}$. In the 200-300 nm region, there is one strong UV line at $297 \mathrm{~nm}$ and five excited nitride oxide (NO) lines at 226, 236, 246, 270, and $283 \mathrm{~nm}$. Nitrogen lines dominate the 300-400 region with the signatures of the excited $\mathrm{N}_{2}$ species at 316 , $337,354,357,358$, and $380 \mathrm{~nm}$, whereas the $\mathrm{OH}$ lines are seen at 309, 614, and $616 \mathrm{~nm}$. Oxygen lines are the most numerous. Apart from the metastable oxygen molecule line at $760 \mathrm{~nm}$, there are at least seven atomic oxygen lines at $615,645,700,725,777,794$, and $844 \mathrm{~nm}$. The underpinning plasma chemistry is therefore rather complex.

To study possible correlations of the reactive plasma species to the observed protein reduction, optical emission spectra at different rates of the central oxygen flow were measured for comparison with the protein inactivation kinetics of Fig. 4(b). As shown in Fig. 9(a), NO line intensities at 270 and $283 \mathrm{~nm}$ and the UV line intensity at $297 \mathrm{~nm}$ reduce with increasing flow rate of the central $\mathrm{O}_{2}$ flow, and so do not correlate to the $\mathrm{O}_{2}$ rate dependence of the protein reduction in Fig. 4(b). On the other hand, emission intensities of the three short NO lines at 226, 236, and $246 \mathrm{~nm}$ increase with the $\mathrm{O}_{2}$ rate and appear to relate to the trend of the protein reduction in Fig. 4(b). It is in fact intriguing to observe the similar plateaus, over the $10-50 \mathrm{sccm}$ range, in the $\mathrm{O}_{2}$ rate dependence of the $D$ value [see Fig. 4(b)] and in that of the line intensities at the three short NO wavelengths [see Fig. 9(a)]. However, this is contradictory to the results of Fig. 7(b), where the role of UV seems very small. This suggests that nitride oxide may have contributed to the protein reduction of Fig. 4(b) but may have done so after initial protein damage by other plasma species.

As the central oxygen flow rate increased, the optical emission intensities of all excited nitrogen lines at 316, 337, $354,357,380$, and the $\mathrm{N}_{2}^{+}$line at $358 \mathrm{~nm}$ were found to reduce as seen in Fig. 9(b). Therefore, nitrogen metastables and nitrogen ions do not appear to correlate to the protein reduction in Fig. 4. Similarly, the optical emission intensities of the three $\mathrm{OH}$ lines at 309, 614, and $616 \mathrm{~nm}$ have a decreasing dependence on the $\mathrm{O}_{2}$ flow rate in Fig. 9(c), suggesting that $\mathrm{OH}$ radicals are unlikely to play a dominant role in the protein reduction of Fig. 4. Reduction in the emission intensities of the three helium lines $(587,668$, and $706 \mathrm{~nm})$ is a result of an increased breakdown voltage with an increasing oxygen admixture in the working gas and hence a decreased level of gas ionization.

Figure 9(d) shows the trend of the metastable oxygen molecules, $\mathrm{O}_{2}\left({ }^{1} \Sigma_{g}^{+}\right)$, at $760 \mathrm{~nm}$ and the excited oxygen atoms at seven wavelengths (e.g., 615, 645, 700, 725, 777, 794, and $844 \mathrm{~nm})$. Compared to the pure-helium case, for which the central oxygen flow rate is zero, the line intensities at 777 and $844 \mathrm{~nm}$ are stronger in the $5-10 \mathrm{sccm}$ range and the 5-23 sccm of the central $\mathrm{O}_{2}$ flow rate, respectively. All other

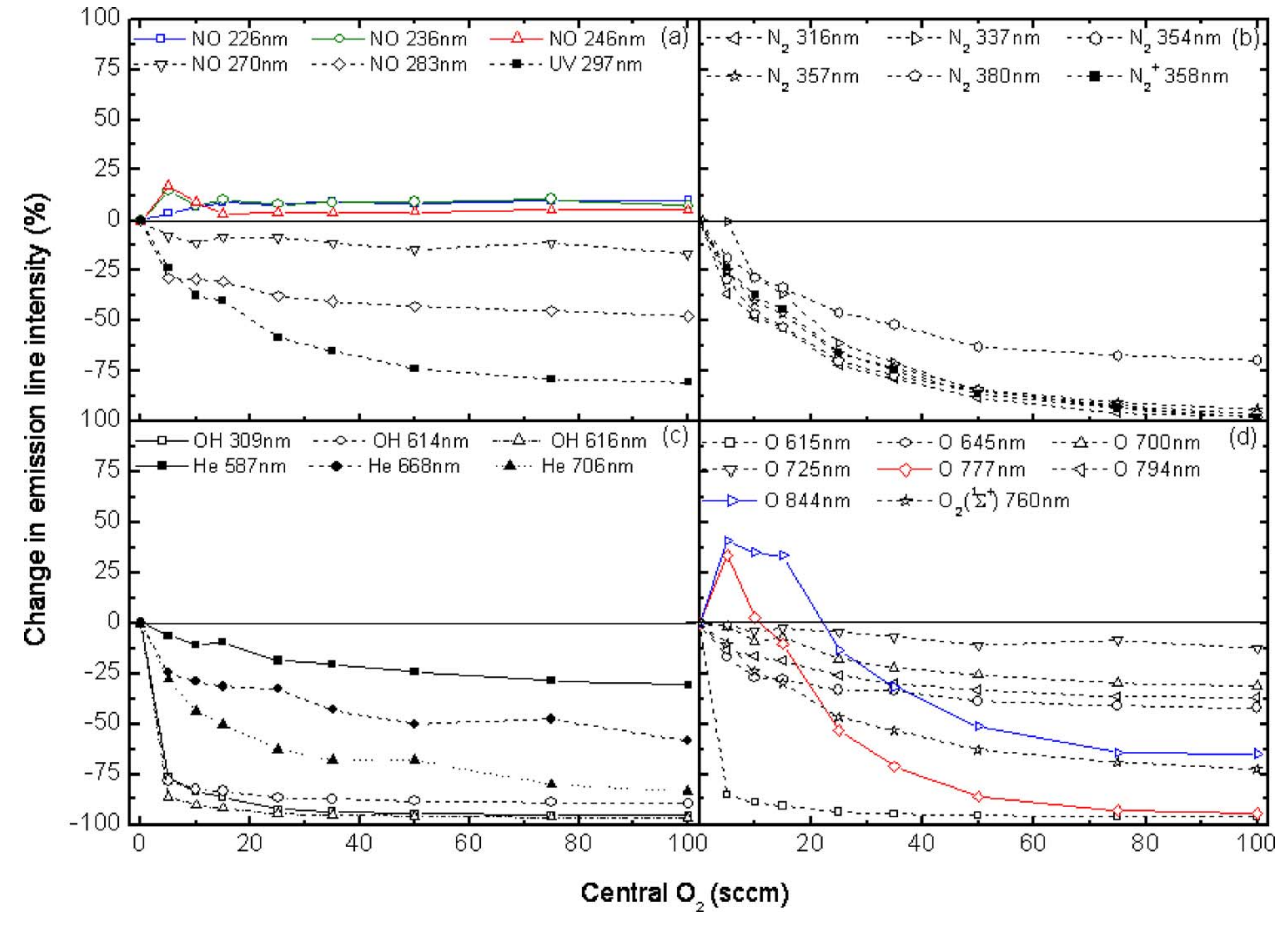

FIG. 9. (Color online) Percentage changes in the line intensities of (a) UV and nitride oxide in the 200-300 $\mathrm{nm}$ region; (b) nitrogen species; (c) $\mathrm{OH}$ radicals and excited helium species; and (d) excited oxygen species, all with $V_{a}=8 \mathrm{kV}$ and no side $\mathrm{O}_{2}$ flow. 
lines in Fig. 9(d) reduce with increasing oxygen flow rate. Therefore, the excited oxygen atoms associated with the emission channels at 777 and $844 \mathrm{~nm}$ have a similar $\mathrm{O}_{2}$ dependence to that of the protein reduction in Fig. 4(b), and as such may be responsible for the observed protein reduction. It is worth emphasizing that the $\mathrm{O}_{2}$ rate range of 5-23 sccm in which the line intensities at 777 and $844 \mathrm{~nm}$ are large in Fig. 9(d) is smaller than the $\mathrm{O}_{2}$ rate range of 5-50 sccm in which the $D$ value is close to its minimum in Fig. 4(b). We also notice that the NO line intensities at 226, 236, and $246 \mathrm{~nm}$ are always stronger than those in the pure helium case over a very large range of $5-100 \mathrm{sccm}$ in the central $\mathrm{O}_{2}$ flow rate. It is therefore probable that protein reduction observed may be caused by both atomic oxygen and nitride oxide. More specifically, we speculate that for the 5-23 sccm range the excited-state oxygen atoms cause an initial degradation to the surface-borne protein and help increase the subsequent damage by the excited nitride oxide species. This synergistic effect is stronger in the $5-15 \mathrm{sccm}$ range in which the dosage of excited NO species is below optimal, with the line intensity at $246 \mathrm{~nm}$ reducing and with the intensity at $226 \mathrm{~nm}$ yet to reach its maximum.

For the $\mathrm{O}_{2}$-rate range of 50-100 sccm, all excited oxygen lines are of lower emission intensities than their values in the pure helium case. Although the three short NO wavelengths are seen in Fig. 9(a) to maintain their levels at 50 $\mathrm{sccm}$, the low protein reduction efficiency above $50 \mathrm{sccm}$ in Fig. 4(b) suggests that protein destruction by NO alone is ineffective. By considering the two $\mathrm{O}_{2}$-rate ranges of 5-23 and 50-100 sccm, the comparison of optical emission spectra and protein reduction kinetics supports strongly a synergistic effect between atomic oxygen and excited nitride oxide. This synergistic effect is further supported by the sharp minimum of the final protein residue at $25 \mathrm{sccm}$, even though the $\mathrm{O}_{2}$ rate of $25 \mathrm{sccm}$ is just outside the $5-23 \mathrm{sccm}$ range. It is worth mentioning that the correlation between optical emission spectra and protein reduction kinetics is less obvious for atomic oxygen and excited nitride oxide in the $23-50 \mathrm{sccm}$ range. Therefore, other reactive plasma species not shown in Figs. 8 and 9 may have also contributed to the persistently low $D$ value in the $23-50 \mathrm{sccm}$ range and to the sharp minimum of the final protein residue at $25 \mathrm{sccm}$. These may include ground-state atomic oxygen, ground-state nitride oxide, and ozone. We are currently developing their measurement techniques and will report their effects in a future note.

\section{CONCLUDING REMARKS}

In this contribution, we have attempted to answer two questions-whether atmospheric pressure glow discharges can destruct surface-borne proteins and, if so, what the protein destruction mechanisms may be. By using a heliumoxygen APGD jet, we have shown that surface protein can be reduced significantly. Electrophoresis experiments reported in our previous study suggest that protein residue left on the stainless-steel surface after APGD treatment has sustained considerable degradation. ${ }^{14}$ It is conceivable that protein removed from the stainless-steel surface by APGD treatment is likely to sustain even greater damage. Therefore, protein re-

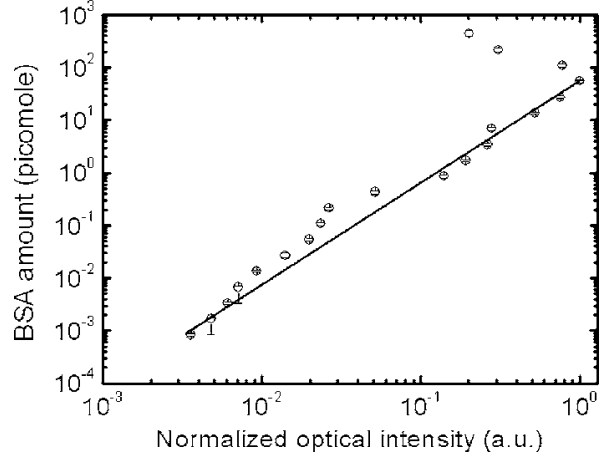

FIG. 10. Linear correlation of surface protein on fluorescence light intensity with improved emission and dichroic filters introduced to the LIF system of Fig. 2(a).

duction observed is through destruction and degradation. Even if plasma treatment cannot completely remove all proteinaceous matters from stainless-steel surfaces, the plasmatreated but unremoved proteins are likely to have compromised integrity and possibly reduced infectivity, thus posing less risk than untreated proteins. Clearly, this adds a significant benefit to the capability of any APGD-based protein inactivation technology.

Kinetics of APGD protein reduction is typically biphasic, strongly affected by the thickness profile of the surface protein. It is however worth mentioning that the time scale of the initial phase of rapid protein reduction is shorter than the time scale over which the central region of the protein deposit is removed (see Fig. 6). This indicates the possibility of an additional factor, for example from the different time scales at which different plasma species damage the surface protein. Using the LIF protein detection system of Fig. 2 with a detection limit of 6.7 femtomoles, the final protein residue left on the stainless-steel surface was 35 femtomoles [see Fig. 4(b)], about $3 \log$ reductions from an initial value of 55 picomoles. This minimum protein residue was found to depend on the protein detection limit. We replaced in the LIF system of Fig. 2(a) the original emission filter (Thorlabs, FEL0500) with an FITC filter (Laser2000, FF506-Em02-25) and the original dichroic filter (Nikon, $505 \mathrm{~nm}$ ) with a Laser2000 filter (FF506-Di02-25x36). The detection limit was found to reduce to 0.84 femtomole as shown in Fig. 10, or 0.17 femtomole $/ \mathrm{mm}^{2}$. With the improved protein detection capability, the minimum protein residue was found to reduce from 35 to 1.8 femtomoles, or some $4.5 \log$ reduction from the initial 55 picomoles. This is equivalent to 0.36 femtomole $/ \mathrm{mm}^{2}$ or $3.6 \times 10^{8}$ molecules $/ \mathrm{mm}^{2}$. With future improvement in the protein detection capability, the minimum protein residue may fall further.

A parallel study on possible physical mechanisms has also been presented. By interplaying protein reduction kinetics with optical emission spectroscopy, atomic oxygen and nitride oxide have been shown to play significant roles, possibly in a synergistic way, in destructing and degrading surface protein. Plasma conditions under which atomic oxygen and excited NO are most abundant have been shown to be similar to plasma conditions under which protein reduction is most effective. Among plasma species not detectable by op- 
tical emission spectroscopy, ground-state atomic oxygen and ground-state NO are likely to have contributed in a notinsignificant fashion and in a way that supports the primary contribution of excited atomic oxygen and excited nitride oxide. This will be discussed in a future article.

\section{ACKNOWLEDGMENTS}

The authors would like to acknowledge the generous financial support of the Department of Health, UK. They are also grateful to the advice of and discussion with Dr. P. R. Richardson, Dr. A. C. Jones, and Dr. J. S. Barton on protein detection techniques.

${ }^{1}$ P. Brown, M. Preece, J. P. Brandel, T. Sato, L. McShane, I. Zerr, A. Fletcher, R. G. Will, M. Pocchiari, N. R. Cashman, J. H. d'Aignaux, L. Cervenakova, J. Fradkin, L. B. Schonberger, and S. J. Collins, Neurology 55, 1075 (2000).

${ }^{2}$ A. Smith, M. Dickson, J. Aitken, and J. Bagg, J. Hosp. Infect. 51, 233 (2002).

${ }^{3}$ J. R. Roth, Industrial Plasma Engineering: Vol. I. Principles (Institute of Physics, Bristol, 1995), pp. 453-461.

${ }^{4}$ M. Laroussi, Plasma Processes Polym. 2, 391 (2005).

${ }^{5}$ K. Kelly-Wintenberg, T. C. Montie, C. Brickman, J. R. Roth, A. K. Carr, K. Sorge, L. C. Wadsworth, and P. P. Y. Tsai, J. Ind. Microbiol. Biotechnol. 20, 69 (1998).

${ }^{6}$ H. W. Herrmann, I. Henins, J. Park, and G. S. Selwyn, Phys. Plasmas 6, 2284 (1999).

${ }^{7}$ N. S. Panikov, S. Paduraru, R. Crowe, P. J. Ricatto, C. Christodoulatos, and K. Becker, IEEE Trans. Plasma Sci. 30, 1424 (2002).

${ }^{8}$ M. Vleugels, G. Shama, X. Deng, E. Greenacre, T. Brocklehurst, and M. G. Kong, IEEE Trans. Plasma Sci. 33, 824 (2005).

${ }^{9}$ J. R. Roth, D. M. Sherman, R. B. Gadri, F. Karakaya, Z. Chen, T. C.
Montie, K. Kelly-Wintenberg, and P. Y. Tsai, IEEE Trans. Plasma Sci. 28, 56 (2000).

${ }^{10}$ W. Lai, H. Lai, S. P. Kuo, O. Tarasenko, and K. Levon, Phys. Plasmas 12, 023501 (2005).

${ }^{11}$ X. T. Deng, J. J. Shi, G. Shama, and M. G. Kong, Appl. Phys. Lett. 87, 153901 (2005).

${ }^{12}$ H. Yu, S. Perni, J. J. Shi, D. Z. Wang, M. G. Kong, and G. Shama, J. Appl. Microbiol. 101, 1323 (2006).

${ }^{13}$ C. Bernard, A. Leduc, J. Barbeau, B. Saoudi, L'H. Yahia, and G. De Crescenzo, J. Phys. D: Appl. Phys. 39, 3470 (2006).

${ }^{14}$ X. T. Deng, J. J. Shi, and M. G. Kong, Appl. Phys. Lett. 90, 013903 (2007).

${ }^{15}$ S. Perni, X. T. Deng, G. Shama, and M. G. Kong, IEEE Trans. Plasma Sci. 34, 1297 (2006)

${ }^{16}$ H. C. Baxter, G. A. Campbell, A. G. Whittaker, A. C. Jones, A. Aitken, A. H. Simpson, M. Casey, L. Bountiff, L. Gibbard, and R. L. Baxter, J. Gen. Virol. 86, 2393 (2005).

${ }^{17}$ X. T. Deng, J. J. Shi, G. Shama, and M. G. Kong, Appl. Phys. Lett. 87, 153901 (2005).

${ }^{18}$ J. L. Walsh, J. J. Shi, and M. G. Kong, Appl. Phys. Lett. 88, 171501 (2006).

${ }^{19}$ V. I. Kovalev, J. S. Barton, P. R. Richardson, and A. C. Jones, presented at 2nd International Conference on Optical and Laser Diagnostics, London, UK, September 2005.

${ }^{20}$ P. R. Richardson, A. C. Jones, R. L. Baxter, H. C. Baxter, A. G. Whittaker, and G. A. Campbell, Proc. SPIE 5502, 291 (2004).

${ }^{21}$ X. T. Deng, J. J. Shi, and M. G. Kong, IEEE Trans. Plasma Sci. 34, 1310 (2006).

${ }^{22}$ M. G. Kong and X. T. Deng, IEEE Trans. Plasma Sci. 31, 7 (2003).

${ }^{23}$ J. J. Shi, X. T. Deng, R. Hall, J. D. Punnett, and M. G. Kong, J. Appl. Phys. 94, 6303 (2003).

${ }^{24}$ J. J. Shi and M. G. Kong, J. Appl. Phys. 94, 5504 (2003).

${ }^{25}$ M. Laroussi, D. A. Mendis, and M. Rosenberg, New J. Phys. 5, 41 (2003).

${ }^{26}$ P. R. Chalise, S. Perni, G. Shama, B. M. Novac, I. R. Smith, and M. G. Kong, Appl. Phys. Lett. 89, 153902 (2006). 